This item was submitted to Loughborough's Research Repository by the author.

Items in Figshare are protected by copyright, with all rights reserved, unless otherwise indicated.

\title{
Exercise intensity and postprandial health outcomes in adolescents
}

PLEASE CITE THE PUBLISHED VERSION

http://dx.doi.org/10.1007/s00421-014-3074-8

\section{PUBLISHER}

(C) Springer

VERSION

AM (Accepted Manuscript)

\section{PUBLISHER STATEMENT}

This work is made available according to the conditions of the Creative Commons Attribution-NonCommercialNoDerivatives 4.0 International (CC BY-NC-ND 4.0) licence. Full details of this licence are available at: https://creativecommons.org/licenses/by-nc-nd/4.0/

\section{LICENCE}

CC BY-NC-ND 4.0

\section{REPOSITORY RECORD}

Bond, Bert, Craig A. Williams, Carly Isic, Sarah R. Jackman, Keith Tolfrey, Laura A. Barrett, and Alan R. Barker. 2019. "Exercise Intensity and Postprandial Health Outcomes in Adolescents". figshare. https://hdl.handle.net/2134/20854. 


\section{Exercise intensity and postprandial health outcomes in adolescents}

Bert Bond ${ }^{1}$, Craig A. Williams ${ }^{1}$, Carly Isic ${ }^{1}$, Sarah R. Jackman ${ }^{2}$, Keith Tolfrey ${ }^{3}$, Laura A. Barrett $^{3}$ and Alan R. Barker ${ }^{1}$

${ }^{1}$ Children's Health and Exercise Research Centre, Sport and Health Science, College of Life and Environmental Sciences, University of Exeter, Exeter, EX1 2LU.

${ }^{2}$ Sport and Health Science, College of Life and Environmental Sciences, University of Exeter, Exeter, EX1 2LU.

${ }^{3}$ Paediatric Exercise Physiology Research Group, School of Sport, Exercise and Health Science, Loughborough University, Loughborough, LE11 3TU.

Corresponding author:

Dr Alan R. Barker

Children's Health and Exercise Research Centre, Sport and Health Sciences

College of Life and Environmental Sciences

University of Exeter

St Luke's Campus

Exeter

EX1 2LU

Tel: $44(0) 1392722766$

Fax: 44 (0)1392 724726

Email: A.R.Barker@exeter.ac.uk

This study was supported by the Sport and Health Sciences Research Committee, College of Life and Environmental Sciences, University of Exeter.

The authors confirm the absence of any conflicts of interest. 


\section{ABSTRACT}

PURPOSE: The effect of exercise intensity and sex on postprandial risk factors for cardiovascular disease in adolescents is unknown. We examined the effect of a single bout of work-matched high-intensity interval exercise (HIIE) and moderate intensity exercise (MIE) on postprandial triacylglycerol (TAG) and systolic blood pressure (SBP) in adolescents. METHOD: Twenty adolescents (10 male, $14.3 \pm 0.3$ y) completed three 1-day trials: 1 ) rest (CON); 2) $8 \times 1$ min cycling at 90\% peak power with 75 s recovery (HIIE); 3) cycling at 90\% of the gas exchange threshold (MIE), one hour before consuming a high fat milkshake (1.50 g fat and $80 \mathrm{~kJ} \mathrm{~kg}^{-1}$ ). Postprandial TAG, SBP and fat oxidation were assessed over four hours. RESULTS: Compared to CON, the incremental area under the curve for TAG (IAUC-TAG) was not significantly lowered in HIIE $(P=0.22$, effect size $(E S)=0.24)$ or $\operatorname{MIE~}(P=0.65$, $E S=0.04)$ for boys. For girls, HIIE and MIE lowered IAUC-TAG by 34\% ( $P=0.02, E S=0.58)$ and $38 \%(P=0.09, E S=0.73)$ respectively, with no difference between HIIE and MIE $(P=0.74, E S=0.14)$. Changes in TAG were not related to energy expenditure during exercise or postprandial fat oxidation. Postprandial SBP (total-AUC pooled for both sexes) was lower in HIIE compared to $\operatorname{CON}(P=0.01, E S=0.68)$ and MIE $(P=0.02, E S=0.60)$, with no difference between MIE and CON ( $P=0.45, E S=0.14)$. CONCLUSION: A single bout of HIIE and MIE, performed one hour before a HFM, can meaningfully attenuate IAUC-TAG in girls but not boys. Additionally, HIIE, but not MIE, may lower postprandial SBP in normotensive adolescents. 


\section{KEY WORDS}

Cardiovascular disease (CVD), high-intensity exercise, triacylglycerol (TAG), young people, physical activity.

\section{ABBREVIATIONS}

95\% CI = 95\% confidence intervals

$\mathrm{CON}=$ Control

CVD = Cardiovascular disease

$\mathrm{EE}=$ Energy expenditure

$\mathrm{ES}=$ Effect size

HFM = High fat meal

HIIE = High-intensity interval exercise

$\mathrm{HR}=$ Heart rate

IAUC = Incremental area under the curve versus time

MIE = Moderate-intensity exercise

PPH $=$ Postprandial hypertension

PPL = Postprandial lipaemia

$\mathrm{RMR}=$ resting metabolic rate

SBP $=$ systolic blood pressure

TAG $=$ triacylglycerol

TAUC $=$ Total area under the curve versus time

$\dot{V} \mathrm{O}_{2}=$ Oxygen consumption 


\section{INTRODUCTION}

Postprandial lipaemia (PPL) has been implicated in the progression of atherosclerosis (Zilversmit 1979), and is an independent predictor of cardiovascular disease (CVD) in adults (Nordestgaard et al. 2007). Although the clinical significance of atherosclerosis is not apparent until later life, the atherosclerotic process has its origins in childhood (Stary 1989) and the progression of which is associated with CVD risk factors in childhood and adulthood (Berenson et al. 1998). Systolic blood pressure (SBP) during adolescence is also associated with future CVD risk (Berenson et al. 1998), and postprandial hypertension (PPH) has been purported as a novel atherosclerotic risk factor in adults (Uetani et al. 2012). Considering that up to two thirds of the day may be spent in the postprandial state, interventions that attenuate PPL and PPH in young people may offer primary prevention against the development of atherosclerosis.

It is known that 60 min of intermittent or continuous exercise at a moderate to vigorous intensity $\left(50-75 \% \dot{V} \mathrm{O}_{2}\right.$ peak) can reduce PPL by 20-30\% in male and female adolescents (Tolfrey et al. 2012; Tolfrey et al. 2008; Tolfrey et al. 2013). Furthermore, $30 \mathrm{~min}$ of moderate exercise ( $<1 \mathrm{MJ}$ ) was similarly effective at reducing PPL as $60 \mathrm{~min}$ in 13 year old boys (Tolfrey et al. 2012), indicating that even a small volume of exercise may have a beneficial effect on PPL. However, the same authors failed to observe a meaningful reduction in PPL after 30 min of moderate exercise in 10-14 year old girls (Tolfrey et al. 2013). Currently, the optimal exercise interventions to modulate PPL in adolescent boys and girls are currently unknown, and we are not aware of a single study that has investigated the effect of exercise on PPH in young people.

Recent evidence has identified that low volume, high-intensity interval exercise (HIIE) can lower PPL in healthy young adults (Freese et al. 2011), and may be more effective than moderate intensity exercise (MIE) (Trombold et al. 2013). Furthermore, an increase in postprandial resting fat oxidation was related to the beneficial effects of HIIE on PPL (Trombold et al. 2013). It has recently been demonstrated that HIIE can lower PPL in 12-13 y old boys (Thackray et al. 2013), but this study did not compare the efficacy of HIIE to a bout of isoenergetic MIE to isolate the effect of exercise intensity. As many children fail to achieve the recommended 60 min of daily moderate intensity physical activity (Riddoch et al. 
2007), it is important to identify whether low volume HIIE offers either similar or superior benefits to postprandial health outcomes compared to traditional MIE.

Surprisingly, no data are available regarding the influence of an acute bout of exercise on PPL in adolescents when exercise is performed immediately before a HFM. Reductions in PPL are possible in adult males when exercise is performed $60 \mathrm{~min}$ before the test meal (Katsanos et al. 2004), however this acute response may be sex dependent (Henderson et al. 2010). Recent evidence in youth suggests that exercise performed during the postprandial period does not influence PPL (Sisson et al. 2013), but it is currently unknown whether exercise performed on the same day prior to the test meal can modulate PPL in young people.

The primary aim of this study was to identify the influence of exercise intensity (workmatched MIE vs. HIIE) performed one hour before a HFM on postprandial plasma [TAG] and SBP in adolescent boys and girls. The secondary aim was to investigate whether changes in PPL were related to changes in resting fat oxidation during the postprandial period.

\section{METHOD}

\section{Participants}

Twenty 13 to 14-year-old adolescents (10 girls) volunteered to take part in this study. Participant assent and parental consent were obtained before participation in the project, which was approved by the institutional ethics committee. Participants showed no contraindications to exercise and were not using any medication or substance known to influence carbohydrate or fat metabolism.

Body mass, seated height and stature were measured to the nearest $0.1 \mathrm{~kg}$ and $0.1 \mathrm{~cm}$ respectively. Percentage body fat was estimated using triceps and subscapular skinfold thickness according to Slaughter et al. (Slaughter et al. 1988) and pubertal status was determined by a self-assessment of secondary sexual characteristics using adapted drawing of the five Tanner stages of public hair development (Morris 1980).

\section{Experimental protocol}


This study required four visits to the laboratory, separated by approximately 1 week, and incorporated a within measures design. All exercise tests were performed using an electronically braked cycle ergometer (Lode Excalibur Sport, Groningen, the Netherlands).

\section{Visit 1: $\dot{V} O_{2 \max }$ and gas exchange threshold determination}

Participants were habituated to exercise on the cycle ergometer before completing a combined ramp and supramaximal test to exhaustion to establish $\dot{V} \mathrm{O}_{2 \text { max }}$ (Barker et al. 2011). Pulmonary $\dot{V} \mathrm{O}_{2}$ was monitored throughout (Cortex Metalyzer III B, Leipzig, Germany) and the gas exchange threshold (GET) was identified as the disproportionate increase in carbon dioxide production $\left(\dot{V} \mathrm{CO}_{2}\right)$ relative to $\dot{V} \mathrm{O}_{2}$ and an increase in expired ventilation $(\dot{V} \mathrm{E}) / \dot{V} \mathrm{O}_{2}$ with no increase in $\dot{V} \mathrm{E} / \dot{V} \mathrm{CO}_{2} . \dot{V} \mathrm{O}_{2}$ max was determined as the highest 10 second average in $\dot{V} \mathrm{O}_{2}$ elicited either during the ramp test or supramaximal bout.

\section{Visits 2-4: Exercise and postprandial measures}

An overview of the study protocol is illustrated in Figure 1. Following a $\sim 12$ h overnight fast, participants arrived at the laboratory at 07:45 and rested for 10 min before providing a fasting capillary blood sample for plasma [TAG] and [glucose]. Blood pressure was determined as the mean of three measures using an automated inflation cuff (Dinamap Carescape V100, GE Healthcare, USA). Participants were seated with their backs supported and feet on the ground for 10 min before the assessment of blood pressure, and remained silent throughout the measures. Resting metabolic rate (RMR) was assessed at 08:15 via indirect calorimetry (Cortex Metalyzer II, Leipzig, Germany) for $15 \mathrm{~min}$ in order to determine total energy expenditure (EE) and substrate oxidation (fat and carbohydrate) following each trial. RMR was identified as the average $\dot{V} \mathrm{O}_{2}$ after the removal of errant $\dot{V} \mathrm{O}_{2}$ values lying more than 4 standard deviations (SD) from the local mean. Between 08:30 and 08:45 participants consumed a standard breakfast cereal with $125 \mathrm{~mL}$ semi skimmed milk (2.5 g fat, $31 \mathrm{~g}$ carbohydrates, 6 g protein, $732 \mathrm{~kJ}$ energy intake).

At 09:45, $1 \mathrm{~h}$ after breakfast, participants completed on separate days and in a randomised order: 1) 23 min of HIIE; 2) 30 min of continuous moderate intensity cycling at 90\% GET (MIE); or 3) rested in the laboratory for $30 \mathrm{~min}$. The HIIE bout consisted of a 3 min warm up at $20 \mathrm{~W}$, followed by $8 \times 1 \mathrm{~min}$ intervals at $90 \%$ of the peak power determined from the ramp test to exhaustion, interspersed with $75 \mathrm{~s}$ of recovery at $20 \mathrm{~W}$, before a $2 \mathrm{~min}$ cool down at 20 
W. The duration of the MIE trial was calculated to match the total external work performed during the HIIE bout for each participant. Heart rate (HR), $\dot{V} \mathrm{O}_{2}$ and $\dot{V} \mathrm{CO}_{2}$ were monitored throughout both trials. For both MIE and HIIE the participants provided a rating of perceived exertion (RPE) using the 1-10 Pictorial Children's Effort Rating Table (Yelling M. 2002) in the final 10 s of exercise, and then completed the 16-point Physical Activity Enjoyment Scale (PACES) (Motl et al. 2001) on completion of the exercise. After their final exercise trial, each participant was asked to identify which exercise bout they preferred. Exercise was completed by 10:15.

One hour after the completion of the rest/exercise condition, post exercise plasma [TAG], [glucose] and blood pressure were assessed. Participants then consumed a milkshake of 3 parts Cornish ice cream and 1 part double cream between 11:15 and 11:30, which provided $1.50 \mathrm{~g}$ of fat (70\% total energy), $1.20 \mathrm{~g}$ carbohydrate (25\%) and $0.21 \mathrm{~g}$ of protein (5\%) per kilogram of body mass $\left(80 \mathrm{~kJ} \cdot \mathrm{kg}^{-1}\right)$ in accordance with previous postprandial investigations in this population (Tolfrey et al. 2012; Tolfrey et al. 2013). Plasma [TAG], [glucose], blood pressure, RMR and substrate oxidation were assessed at hourly intervals during the $4 \mathrm{~h}$ postprandial period. A $4 \mathrm{~h}$ postprandial observational period was employed as this has been shown to provide a valid estimate of the PPL response compared to an $8 \mathrm{~h}$ observational period (Weiss et al. 2008). No other food was consumed during the postprandial period, although water was available ad libitum and subsequently replicated for each trial. Participants remained in the laboratory and inactive throughout the postprandial period, by reading, completing homework, watching DVDs or playing computer games.

\section{Blood analyses}

For each blood sample, $\sim 600 \mu \mathrm{L}$ of capillary blood was collected into lithium-heparin coated ([TAG]) and heparin-fluoride coated ([glucose]) Microvette CB 300 tubes (Sarstedt Ltd, Leicester, UK) and centrifuged immediately at 13,000 g for $15 \mathrm{~min}$. Plasma was then removed and either stored at $-80^{\circ} \mathrm{C}$ for 1 month for [TAG] analysis, or analysed immediately for [glucose] (YS1 2300 Stat Plus Glucose and L-Lactate Analyzer, YSI Inc., Yellow Springs, USA). Plasma [TAG] was quantified in duplicate by enzymatic, colorimetric methods using an assay kit according to the manufacturer's guidelines (Cayman Chemical Company, MI, USA). The within-batch coefficients of variation for plasma [TAG] and [glucose] were 2.9 and $1.0 \%$ respectively. Haematocrit and haemoglobin values were 
determined from the fasted and final capillary blood samples in order to calculate plasma volume. Changes in plasma volume were small across each trial (-2.6 to $1.7 \%)$.

\section{Calculation of RMR and substrate oxidation}

Total EE and the contributions of fat and carbohydrate oxidation to MIE were estimated using the mean exercise $\dot{V} \mathrm{O}_{2}$ and respiratory exchange ratio (RER) for each 15 min measurement period (Frayn 1983). Protein oxidation was assumed to be negligible, and an RER $>1$ during exercise was taken to represent $100 \%$ carbohydrate oxidation.

\section{Standardisation of diet and exercise}

With parental supervision, participants were asked to complete a food diary during the $48 \mathrm{~h}$ period immediately preceding each laboratory visit. Participants were asked to replicate their diet prior to each laboratory visit and were verbally reminded of this requirement. The food diaries were subsequently assessed for total energy and macronutrient intake (CompEat Pro, Nutrition Systems, UK). Participants were also asked to avoid strenuous exercise during this period.

\section{Statistical analyses}

Area under the curve (AUC) analyses were performed using the trapezium rule (GraphPad Prism, GraphPad Software, San Diego, CA) to describe the changes in plasma [TAG], [glucose], SBP, RMR and fat oxidation over the $4 \mathrm{~h}$ period following the HFM. Both total (TAUC) and incremental (IAUC) analyses were performed to characterise the magnitude of the response and the changes over time respectively. It has previously been shown that changes in TAUC-TAG are largely attributable to differences in baseline [TAG] after exercise (Kolifa et al. 2004), and that IAUC-TAG more accurately describes the [TAG] response after a test meal (Carstensen et al. 2003). Consequently, the IAUC analysis was used to account for changes in baseline plasma [TAG] across the experimental conditions prior to the HFM and adopted as our primary outcome measure which is in line with previous studies (Petitt et al. 2003; Trombold et al. 2013). All AUC analyses were calculated using the time point immediately before the HFM (baseline).

Descriptive statistics were calculated using SPSS (version 19.0, Chicago, USA) and presented as mean \pm SD. Mean differences in descriptive statistics between boys and girls 
were analysed using independent samples $t$ tests. The mean differences in the physiological and perceptual responses of the boys and girls during HIIE and MIE were analysed using paired samples $t$ tests. Analysis of fasting TAG and glucose, and AUC analyses for TAG, glucose, fat oxidation and SBP were performed using a mixed model ANOVA with trial (CON, MIE, HIIE) and sex (male, female) as the main effects. Normality of distribution was checked using the Shapiro-Wilk test, and data were log transformed if this assumption was violated. Homogeneity of variance was determined using Mauchly's test of sphericity and the degrees of freedom were adjusted using the Greenhouse-Geisser correction if required. To facilitate comparison with recent studies examining exercise and PPL in adolescents (Thackray et al. 2013; Tolfrey et al. 2013) pairwise comparisons between means were interpreted using the $P$ value, 95\% confidence intervals (CI) and standardised effect sizes (ES) (Cohen 1988; Hopkins et al. 2009). The null hypothesis was rejected at an alpha level of 0.05 , and an $E S$ of 0.20 was considered to be a small change between means, with 0.50 and 0.80 interpreted as a moderate and large change respectively (Cohen 1988). Relationships between changes in AUC outcomes for TAG and potentially mechanistically important variables (e.g. postprandial resting fat oxidation) were explored using Pearson's correlation coefficients and their associated 95\% CI.

\section{RESULTS}

Baseline participant characteristics are presented in Table 1. Girls and boys were matched for age and body mass, but boys were taller, had a lower percentage of body fat and higher $\dot{V} \mathrm{O}_{2}$ max compared to girls. The sexual maturation status for boys and girls was as follows; Tanner stage 2, $n=2$ and $n=0$; Tanner stage $3, n=0$ and $n=2$; Tanner stage, $4 n=6$ and $n=5$; Tanner stage $5, n=2$ and $n=3$. No differences in energy intake (main effect for trial, $P=0.98$; main effect for sex, $P=0.17$; trial by sex interaction, $P=0.99$ ) or individual macronutrient contributions (main effects for trials all $P>0.70$; main effects for sex all $P>0.71$; trial by sex interaction, $P>0.58$ ) were apparent for boys or girls during the $48 \mathrm{~h}$ preceding each laboratory visit (data not reported).

\section{Exercise conditions}

Table 2 presents the physiological and perceptual data from the exercise trials. Despite no differences in work done, HIIE elicited a higher $\dot{V} \mathrm{O}_{2}$, heart rate and RPE compared to MIE for both boys and girls. The highest $\dot{V} \mathrm{O}_{2}$ achieved during the HIIE condition equated to $93 \pm$ 
5\% and $96 \pm 4 \% \dot{V} \mathrm{O}_{2 \text { max }}$ for boys and girls respectively. Average length of the MIE trial was $27.1 \pm 3.5$ min. Enjoyment, as measured using PACES was higher for HIIE for boys and girls, and seven boys and seven girls indicated that they preferred the HIIE exercise bout.

\section{Plasma [TAG]}

There were no differences in fasted plasma [TAG] between trials for boys or girls (main effect for trial, $P=0.86$; main effect for sex, $P=0.59$; trial by sex interaction, $P=0.70$ ). Changes in plasma [TAG] during the postprandial period are illustrated in Figure 2 and the AUC analyses are described in Table 3. No differences were apparent in TAUC-TAG (main effect for trial, $P=0.50$; main effect for sex, $P=0.82$; trial by sex interaction, $P=0.47$ ). However, there was a significant trial by sex interaction for the IAUC-TAG $(P=0.02)$. For boys, IAUC-TAG was not significantly different in HIIE compared with CON ( $P=0.22,95 \%$ CI -0.14 to $0.56, E S=0.24)$ or MIE ( $P=0.34,95 \%$ CI -0.19 to $0.54, E S=0.20)$, or for MIE compared with CON ( $P=0.65$, 95\% CI -0.30 to $0.37, E S=0.04)$. For girls, IAUC-TAG was $34 \%$ lower after MIE compared with CON ( $P=0.02,95 \%$ CI -1.43 to $-0.17, E S=0.58)$ and a strong trend for a 38\% reduction in IAUC-TAG was observed after HIIE compared to CON ( $P=0.09,95 \%$ CI -2.13 to $0.31, E S=0.73$ ). There was no difference between HIIE and MIE for IAUC-TAG $(P=0.74,95 \% \mathrm{CI}-0.77$ to $0.57, E S=0.14)$. There were no differences in IAUC-TAG between boys and girls for $\operatorname{CON}(P=0.52,95 \% \mathrm{CI}-0.91$ to $1.69, E S=0.29)$ or MIE ( $P=0.28,95 \%$ CI -1.28 to $0.40, E S=0.50)$, however IAUC-TAG was lower in girls for HIIE ( $P=0.03,95 \%$ CI -1.36 to $-0.08, E S=1.10)$. Pearson's correlations did not reveal any relationships between percentage body fat, EE or mean $\dot{V} \mathrm{O}_{2}$ and IAUC-TAG for boys or girls (all $r<0.2, P>0.05)$ ).

\section{Plasma [Glucose]}

No differences in fasted plasma [glucose] were apparent for boys or girls between trials (main effect for trial, $P=0.76$; main effect for sex, $P=0.28$; sex by trial interaction, $P=0.72$ ). Changes in plasma [glucose] during the postprandial period are illustrated in Figure 2 and the AUC analyses are presented in Table 3. No differences were present between trials for boys or girls in TAUC-glucose (main effect of trial, $P=0.22$; main effect of sex, $P=0.36$; age by sex interaction, $P=0.44$ ) or IAUC-glucose (main effect of trial, $P=0.89$; main effect of sex, $P=0.56$; age by sex interaction, $P=0.90$ ). 


\section{RMR and fat oxidation}

There was no effect of trial $(P=0.27)$ or trial by sex interaction $(P=0.73)$ on TAUC-RMR, but there was a main effect of sex, which was lower in girls $(P=0.04,95 \%$ CI -228 to -5 , $E S=0.85$, data not presented). There was a main effect for trial on postprandial TAUC-Fat oxidation $(P<0.001)$, but not sex $(P=0.55)$ or a trial by sex interaction $(P=0.63)$. Data were subsequently pooled for further analysis of the TAUC-Fat oxidation main effect $(n=20)$ and are presented in Figure 3. TAUC-Fat oxidation increased in HIIE by $23 \%$ compared to CON $(P<0.001,95 \%$ CI 0.04 to $0.12, E S=0.88)$ and by $16 \%$ compared to MIE $(P=0.001,95 \%$ CI 0.03 to $0.09, E S=0.66$ ). TAUC-Fat oxidation was not different in MIE compared to CON $(P=0.20,95 \%$ CI -0.01 to $0.06, E S=0.28)$. Changes in TAUC-TAG analyses during MIE and HIIE were not related to postprandial TAUC-fat oxidation (all $r<0.2$ ).

\section{Blood pressure and HR}

Changes in TAUC-SBP and HR over time are presented in Figure 3. Compared to the initial fasting measure, SBP was attenuated following HIIE ( $P=0.003$, 95\% CI -8 to $-2, E S=0.72)$ and $\operatorname{MIE}(P=0.04,95 \% \mathrm{CI}-5$ to $0, E S=0.33)$.

There was a main effect for trial on the postprandial TAUC-SBP $(P=0.01)$, but not sex $(P=0.17)$ or a trial by sex interaction $(P=0.39)$. Data were subsequently pooled for further analysis of the TAUC-SBP main effect $(n=20)$ and are presented in Figure 3. Postprandial TAUC-SBP was 3\% lower in HIIE compared to CON ( $P=0.01,95 \%$ CI -19 to $-3, E S=0.68)$ and 3\% lower compared to MIE ( $P=0.02,95 \%$ CI -15 to -2 , $E S=0.60)$. Postprandial TAUCSBP was not different between MIE and CON ( $P=0.45$, 95\% CI -8 to $4, E S=0.14)$. Resting HR was elevated $1 \mathrm{~h}$ post exercise compared to pre exercise values in HIIE, but not MIE, for boys ( $P=0.01,95 \%$ CI 3 to $14, E S=1.13)$ and girls $(P<0.001,95 \%$ CI 7 to $16, E S=1.83)$.

\section{DISCUSSION}

The novel findings of the present study are: 1) based on the IAUC-TAG analyses, both HIIE and MIE reduced PPL (moderate ES) in girls. In contrast, HIIE and MIE did not attenuate PPL in boys; 2) resting postprandial fat oxidation was increased after HIIE compared to CON (large ES) and MIE (moderate ES) for both boys and girls, but was not related to changes in PPL; 3) HIIE reduced postprandial SBP compared to CON and MIE (moderate ES) in boys and girls; and 4) PACES score was greater in HIE compared to MIE for boys and girls (moderate and large ES, respectively). These data, therefore, show for the first time that 
exercise intensity and sex play an important role in modulating different postprandial health outcomes in adolescents when the test meal is consumed one hour after exercise cessation.

There is consistent evidence showing that performing 30-60 min of moderate to vigorous exercise $\left(50-75 \% \dot{V} \mathrm{O}_{2 \text { peak }}\right) \sim 12-16$ h before a HFM can reduce PPL in healthy adolescents (Tolfrey et al. 2014). Furthermore, an acute bout of low volume HIIE running performed 15.5 h before a HFM can reduce TAUC-TAG ( 11\%, ES=0.50) and IAUC-TAG ( 15\%, ES=0.39) over a 6.5 h postprandial period in healthy 11-12 y old boys (Thackray et al. 2013). Given recent data in healthy adult men showing HIIE to be more effectual at attenuating PPL compared to an isoenergetic bout of MIE (Trombold et al. 2013), we reasoned HIIE would offer either a similar or superior attenuation of PPL in adolescents compared to a workmatched bout of MIE. In contrast, the present study indicates that reductions in TAUC-TAG are not apparent when a HFM is consumed 1 hour after an exercise bout. However, interpreting the TAUC-TAG has been criticised due to its dependency on changes in fasting plasma [TAG] (Kolifa et al. 2004). We therefore used the IAUC-TAG to quantify the effect of exercise on plasma [TAG] following the HFM as recommended (Carstensen et al. 2003), which is consistent with other PPL studies (Petitt et al. 2003; Trombold et al. 2013).

In adolescent boys we observed no significant changes in IAUC-TAG following HIIE and MIE. In addition, the ES for this finding was either small (HIIE) or trivial (MIE), suggesting no meaningful effect on the IAUC-TAG outcome for boys. This result is surprising given the well documented beneficial effect of MIE and HIIE exercise on plasma [TAG] following a HFM in adolescent boys (ES range from 0.39 to 1.40) (Thackray et al. 2013; Tolfrey et al. 2008). A possible explanation for this lack of effect in boys in the present study could reside in the adoption of a single day protocol, as PPL may be attenuated to a greater degree when exercise is performed $12 \mathrm{~h}$ compared to $1 \mathrm{~h}$ after exercise in adult males (Zhang et al. 1998), possibly due to a delayed increase in activity of lipoprotein lipase (LPL) (Seip and Semenkovich 1998). Furthermore, it has been shown that 135 min of light walking during a 3 h postprandial period does not attenuate PPL in adolescents (Sisson et al. 2013). The EE of exercise may be important in modulating the lipaemic response (Gill et al. 2002) and may explain our findings in adolescent boys. The current study induced a lower EE ( $650 \mathrm{~kJ})$ than previous investigations in adolescent boys ( 1-2.5 MJ (Tolfrey et al. 2012; Tolfrey et al. 2008), and adult studies which reported a reduction in PPL using a similar 1 day protocol to 
that adopted in the present study (4.6 MJ (Katsanos et al. 2004)). Interestingly, our EE was similar to that used by Pfeiffer et al. $(630 \mathrm{~kJ})$, who failed to observe a reduction in PPL in healthy young men (Pfeiffer et al. 2006), and may indicate that the EE in the present study was insufficient to reduce PPL in boys. The EE explanation, however, cannot account for the work of Thackray et al. (2013) who found low volume HIIE running exercise to attenuate PPL in 11-12 y old boys over a 2 day protocol. This may suggest that the delayed increase in LPL activity is a key determinant of the attenuation in PPL after HIIE, and may account for our findings in adolescent boys.

A novel finding of the present study was that the effect of exercise on PPL was dependent on sex with both MIE and HIIE eliciting moderate reductions (34\% and 38\% respectively) in IAUC-TAG in the adolescent girls only. We did not observe any sex differences in PPL for CON, suggesting that this sexual dimorphism may be mechanistically linked to the exercise bout performed $1 \mathrm{~h}$ before the HFM. Our data are consistent with the work of Henderson et al. (2010) who found that exercise at $45 \%$ and $65 \% \dot{V} \mathrm{O}_{2}$ peak reduced plasma [TAG] $3 \mathrm{~h}$ post exercise in young healthy women but not men. While our study cannot offer any insight into the mechanistic basis of this sex difference in PPL as no meaningful relationship was observed for EE, exercise intensity, RMR or substrate utilisation during or after MIE and HIIE exercise, previous adult studies have suggested an important role for body fat distribution (Couillard et al. 1999), the rate of [TAG] uptake by muscle (Horton et al. 2002), and/or hepatic very low density lipoprotein (VLDL) output (Mittendorfer et al. 2003) and metabolism (Magkos et al. 2007).

We found no meaningful difference between work-matched MIE and HIE to attenuate PPL in adolescent girls. Thus, exercise intensity per se does not appear to determine the magnitude of the reduction in PPL in this population. This finding is not consistent with the recent work of Trombold et al. (2013) who found HIIE (repeated bouts of exercise at $\sim 90 \% \dot{V} \mathrm{O}_{2} \max$ ) to be more effectual at reducing the IAUC-TAG compared to $60 \mathrm{~min}$ of moderate intensity exercise $\left(\sim 50 \% \dot{V} \mathrm{O}_{2}\right.$ max $)$ in healthy men using a 2 day protocol. However, a direct comparison between studies is limited due to the confounding effect of sex (Henderson et al. 2010), and the probable disparity in mechanisms underlying the postprandial response after exercise between a 1- and 2-day protocol (Zhang et al. 1998). 
Recent evidence in adults implicates postprandial hypertension as a novel risk factor for atherosclerosis (Uetani et al. 2012). In the present study the HFM promoted a transient increase ( $4 \mathrm{mmHg}$ ) in SBP in the CON trial in both boys and girls, which may be indicative of the endothelial dysfunction and arterial stiffness that has been reported following a HFM (Vogel et al. 1997). Importantly, a significant reduction in postprandial SBP was present after HIIE compared to both CON (ES=0.68) and MIE ( $E S=0.60)$, highlighting for the first time, the role that HIIE can play in modulating postprandial SBP even in normotensive youth. A protective effect afforded by exercise on endothelial function after a HFM has been demonstrated in adolescents (Sedgwick et al. 2012), suggesting that the exercise performed in the current study may have preserved endothelial function. In addition, we observed an increase in resting HR after both exercise trials, suggesting the reduction in SBP may be related to a fall in peripheral vascular resistance via an attenuated sympathetic drive. While it cannot be ruled out that the effect of exercise on SBP in the current study was related, in part, to the hypotensive response observed after exercise cessation, adult data indicates that postprandial SBP remains lower the day after exercise (Miyashita et al. 2008).

Both HIIE and MIE increased postprandial fat oxidation despite no change in RMR in adolescent boys and girls, with HIIE being more effectual than MIE. This is of importance given the relationship between elevated resting fat oxidation and exercise-induced fat loss (Barwell et al. 2009). Similar changes in resting fat oxidation have been reported $24 \mathrm{~h}$ after a single bout of HIIE in overweight and obese men (Whyte et al. 2012), and it has been shown that $\sim 2 \mathrm{MJ}$ of exercise at an intensity corresponding to peak fat oxidation ( $\sim 63 \% \dot{V} \mathrm{O}_{2}$ peak $)$ increases postprandial fat oxidation in normal weight $12 \mathrm{y}$ old girls on the subsequent day (Zakrzewski and Tolfrey 2012). Given the greater increase in postprandial fat oxidation following HIIE in the current study, exercise intensity appears to be an important mediator of this response and offers a low volume alternative to MIE.

The data presented in the current study should be viewed in the light of a number of methodological considerations. Firstly, given the inherent problems in calculating EE via indirect calorimetry during HIIE, we matched the exercise trials based on the mechanical work done. Consequently, substrate oxidation and EE were only determined during MIE in the present study. We also adopted indirect calorimetry to calculate changes in postprandial RMR and substrate oxidation after the exercise bouts, as disturbances to the bicarbonate pool 
have been reported to return to baseline 30 minutes after 6 minutes of high-intensity excise (Stringer et al. 1992), which broadly corresponds with the HIIE stimulus in the present study. Secondly, our study design included a high carbohydrate breakfast which was not standardised to body mass and may have altered the postprandial response to the HFM (Pedersen et al. 1999). However, no differences in mean body mass were apparent between boys and girls indicating the caloric intake relative to size was equivalent across the groups. Thirdly, PPL in adolescents is known to be influenced by exercise performed up to $16 \mathrm{~h}$ before the HFM (Tolfrey et al. 2014). While we are not able to provide objective measurements of our participants' physical activity, all participants were asked not to undertake formal exercise $48 \mathrm{~h}$ before each laboratory visit. Finally, the PACES scale has been validated for use with adolescent girls (Motl et al. 2001), but no study has addressed whether it is appropriate to use with adolescent boys.

\section{CONCLUSIONS}

We have shown that different postprandial health outcomes are dependent on exercise intensity and sex in healthy adolescents when exercise is performed $1 \mathrm{~h}$ before a HFM. Specifically, reductions in PPL were achieved after a single bout of MIE and HIE in girls but not boys, and not dependent on exercise intensity. In contrast, favourable changes in postprandial SBP and lipid oxidation are possible after HIIE, but not MIE, even in normotensive adolescents. Given that PPL is implicated in the atherosclerotic process (Zilversmit 1979), which starts in childhood (Stary 1989), and that SBP is associated with future CVD risk (Berenson et al. 1998), these findings may have clinical significance. Finally, given that HIIE was perceived to be more enjoyable than MIE, despite the greater physiological stress, our findings support the use of HIIE as an attractive, feasible and effective strategy to improve postprandial health outcomes in adolescents.

\section{ACKNOWLEDGEMENTS}

We thank the staff and participants at Exmouth Community College, Devon, UK for their participation in this project.

\section{FUNDING}

This study was supported by the Sport and Health Sciences Research Committee, College of Life and Environmental Sciences, University of Exeter. 


\section{DISCLOSURE STATEMENT}

The authors confirm the absence of any conflicts of interest.

\section{AUTHOR CONTRIBUTIONS}

$\mathrm{BB}, \mathrm{AB}$, and $\mathrm{CW}$ designed the study. $\mathrm{BB}$ and $\mathrm{CI}$ conducted the research. $\mathrm{BB}, \mathrm{SJ}$ and $\mathrm{AB}$ analysed the data. BB and AB wrote the initial draft of the manuscript. All authors edited the manuscript and approved the final draft.

\section{REFERENCES}

Barker AR, Williams CA, Jones AM, Armstrong N (2011) Establishing maximal oxygen uptake in young people during a ramp cycle test to exhaustion $\mathrm{Br} \mathrm{J}$ Sports Med 45:498-503

Barwell ND, Malkova D, Leggate M, Gill JM (2009) Individual responsiveness to exerciseinduced fat loss is associated with change in resting substrate utilization Metabolism 58:1320-1328

Berenson GS, Srinivasan SR, Bao W, Newman WP, 3rd, Tracy RE, Wattigney WA (1998) Association between multiple cardiovascular risk factors and atherosclerosis in children and young adults. The Bogalusa Heart Study N Engl J Med 338:1650-1656

Carstensen M, Thomsen C, Hermansen K (2003) Incremental area under response curve more accurately describes the triglyceride response to an oral fat load in both healthy and type 2 diabetic subjects Metabolism 52:1034-1037

Cohen J (1988) Statistical Power Analysis for the Behavioural Sciences. Lawrence Erlbaum, Hillsdale

Couillard C et al. (1999) Gender difference in postprandial lipemia : importance of visceral adipose tissue accumulation Arterioscler Thromb Vasc Biol 19:2448-2455

Frayn KN (1983) Calculation of substrate oxidation rates in vivo from gaseous exchange $\mathrm{J}$ Appl Physiol 55:628-634

Freese EC, Levine AS, Chapman DP, Hausman DB, Cureton KJ (2011) Effects of acute sprint interval cycling and energy replacement on postprandial lipemia J Appl Physiol 111:1584-1589

Gill JM, Herd SL, Hardman AE (2002) Moderate exercise and post-prandial metabolism: issues of dose-response J Sports Sci 20:961-967

Henderson GC, Krauss RM, Fattor JA, Faghihnia N, Luke-Zeitoun M, Brooks GA (2010) Plasma triglyceride concentrations are rapidly reduced following individual bouts of endurance exercise in women Eur J Appl Physiol 109:721-730

Hopkins WG, Marshall SW, Batterham AM, Hanin J (2009) Progressive statistics for studies in sports medicine and exercise science Med Sci Sports Exerc 41:3-13

Horton TJ, Commerford SR, Pagliassotti MJ, Bessesen DH (2002) Postprandial leg uptake of triglyceride is greater in women than in men Am J Physiol Endocrinol Metab 283:E1192-1202 
Katsanos CS, Grandjean PW, Moffatt RJ (2004) Effects of low and moderate exercise intensity on postprandial lipemia and postheparin plasma lipoprotein lipase activity in physically active men J Appl Physiol 96:181-188

Kolifa M, Petridou A, Mougios V (2004) Effect of prior exercise on lipemia after a meal of moderate fat content Eur J Clin Nutr 58:1327-1335

Magkos F, Patterson BW, Mohammed BS, Klein S, Mittendorfer B (2007) Women produce fewer but triglyceride-richer very low-density lipoproteins than men J Clin Endocrinol Metab 92:1311-1318

Mittendorfer B, Patterson BW, Klein S (2003) Effect of sex and obesity on basal VLDLtriacylglycerol kinetics Am J Clin Nutr 77:573-579

Miyashita M, Burns SF, Stensel DJ (2008) Accumulating short bouts of brisk walking reduces postprandial plasma triacylglycerol concentrations and resting blood pressure in healthy young men Am J Clin Nutr 88:1225-1231

Morris NM, Udry JR (1980) Validation of a self-administered instrument to assess stage of adolescent development Journal of youth and adolescence 9:271-280

Motl RW, Dishman RK, Saunders R, Dowda M, Felton G, Pate RR (2001) Measuring enjoyment of physical activity in adolescent girls Am J Prev Med 21:110-117

Nordestgaard BG, Benn M, Schnohr P, Tybjaerg-Hansen A (2007) Nonfasting triglycerides and risk of myocardial infarction, ischemic heart disease, and death in men and women JAMA 298:299-308

Pedersen A, Marckmann P, Sandstrom B (1999) Postprandial lipoprotein, glucose and insulin responses after two consecutive meals containing rapeseed oil, sunflower oil or palm oil with or without glucose at the first meal Br J Nutr 82:97-104

Petitt DS, Arngrimsson SA, Cureton KJ (2003) Effect of resistance exercise on postprandial lipemia J Appl Physiol 94:694-700

Pfeiffer M, Wenk C, Colombani PC (2006) The influence of 30 minutes of light to moderate intensity cycling on postprandial lipemia Eur J Cardiovasc Prev Rehabil 13:363-368

Riddoch CJ et al. (2007) Objective measurement of levels and patterns of physical activity Arch Dis Child 92:963-969

Sedgwick MJ, Morris JG, Nevill ME, Tolfrey K, Nevill A, Barrett LA (2012) Effect of exercise on postprandial endothelial function in adolescent boys Br J Nutr:1-9

Seip RL, Semenkovich CF (1998) Skeletal muscle lipoprotein lipase: molecular regulation and physiological effects in relation to exercise Exerc Sport Sci Rev 26:191-218

Sisson S, Anderson AE, Short KR, Gardner AW, Whited T, Robledo C, Thompson DM (2013) Light Activity Following a Meal and Post-Prandial Cardiometabolic Risk in Adolescents Pediatr Exerc Sci

Slaughter MH, Lohman TG, Boileau RA, Horswill CA, Stillman RJ, Van Loan MD, Bemben DA (1988) Skinfold equations for estimation of body fatness in children and youth Hum Biol 60:709-723

Stary HC (1989) Evolution and progression of atherosclerotic lesions in coronary arteries of children and young adults Arteriosclerosis 9:I19-32

Stringer W, Casaburi R, Wasserman K (1992) Acid-base regulation during exercise and recovery in humans $\mathrm{J}$ Appl Physiol 72:954-961

Thackray AE, Barrett LA, Tolfrey K (2013) Acute High-Intensity Interval Running Reduces Postprandial Lipemia in Boys Med Sci Sports Exerc

Tolfrey K, Bentley C, Goad M, Varley J, Willis S, Barrett L (2012) Effect of energy expenditure on postprandial triacylglycerol in adolescent boys Eur J Appl Physiol 112:23-31 
Tolfrey K, Doggett A, Boyd C, Pinner S, Sharples A, Barrett L (2008) Postprandial triacylglycerol in adolescent boys: a case for moderate exercise Med Sci Sports Exerc 40:1049-1056

Tolfrey K, Engstrom A, Murphy C, Thackray A, Weaver R, Barrett LA (2013) Exercise Energy Expenditure and Postprandial Lipaemia in Girls Med Sci Sports Exerc

Tolfrey K, Thackray AE, Barrett LA (2014) Acute exercise and postprandial lipemia in young people Pediatr Exerc Sci 26:127-137

Trombold JRPD, Christmas KM, Machin DR, Kim IY, Coyle EF (2013) Acute HighIntensity Endurance Exercise is More Effective than Moderate-Intensity Exercise for Attenuation of Postprandial Triglyceride Elevation J Appl Physiol

Uetani E et al. (2012) Postprandial hypertension, an overlooked risk marker for arteriosclerosis Atherosclerosis 224:500-505

Vogel RA, Corretti MC, Plotnick GD (1997) Effect of a single high-fat meal on endothelial function in healthy subjects Am J Cardiol 79:350-354

Weiss EP, Fields DA, Mittendorfer B, Haverkort MA, Klein S (2008) Reproducibility of postprandial lipemia tests and validity of an abbreviated 4-hour test Metabolism 57:1479-1485

Whyte LJ, Ferguson C, Wilson J, Scott RA, Gill JM (2012) Effects of single bout of very high-intensity exercise on metabolic health biomarkers in overweight/obese sedentary men Metabolism

Yelling M. LKaSI (2002) Validity of a Pictorial Perceived Exertion Scale for Effort Estimation and Effort Production During Stepping Exercise in Adolescent Children European Physical Education Review 8:157-175

Zakrzewski JK, Tolfrey K (2012) Acute effect of Fatmax exercise on the metabolism in overweight and nonoverweight girls Med Sci Sports Exerc 44:1698-1705

Zhang JQ, Thomas TR, Ball SD (1998) Effect of exercise timing on postprandial lipemia and HDL cholesterol subfractions J Appl Physiol 85:1516-1522

Zilversmit DB (1979) Atherogenesis: a postprandial phenomenon Circulation 60:473-485 
Table 1: Participant characteristics

\begin{tabular}{lcccc}
\hline & Boys $(n=10)$ & Girls $(n=10)$ & $95 \%$ CI & ES \\
\hline Age $(\mathrm{y})$ & $14.3 \pm 0.3$ & $14.3 \pm 0.3$ & -0.4 to 0.2 & 0.00 \\
Body mass $(\mathrm{kg})$ & $55.7 \pm 10.5$ & $56.1 \pm 8.8$ & -8.7 to 9.6 & 0.04 \\
Stature $(\mathrm{m})$ & $1.70 \pm 0.09$ & $1.63 \pm 0.04$ & 0.01 to 0.14 & 1.12 \\
Body fat $(\%)$ & $15 \pm 2$ & $25 \pm 4$ & -14 to -8 & 3.89 \\
$\dot{V} \mathrm{O}_{2 \max }\left(\mathrm{L} \cdot \mathrm{min}^{-1}\right)$ & $2.66 \pm 0.56$ & $1.89 \pm 0.27$ & 0.36 to 1.18 & 1.75 \\
$\dot{V} \mathrm{O}_{2 \max }\left(\mathrm{mL}^{-1} \min ^{-1} \cdot \mathrm{kg}^{-1}\right)$ & $47.7 \pm 4.0$ & $34.1 \pm 5.0$ & 9.3 to 17.7 & 3.04 \\
$\mathrm{GET}\left(\mathrm{L} \cdot \mathrm{min}^{-1}\right)$ & $1.34 \pm 0.28$ & $1.08 \pm 0.13$ & 0.06 to 0.47 & 1.19 \\
GET $\left(\% \dot{V} \mathrm{O}_{2 \max }\right)$ & $49 \pm 7$ & $57 \pm 8$ & -14.5 to -1.3 & 1.06 \\
\hline
\end{tabular}

PHV, peak height velocity; HR, heart rate; $\dot{V} \mathrm{O}_{2}$, oxygen uptake; GET, gas exchange threshold

Data presented as mean $\pm \mathrm{SD}$.

$95 \% \mathrm{CI}=95 \%$ confidence interval for the true difference

$\mathrm{ES}=$ effect size 
Table 2: Physiological and perceptual responses to MIE and HIIE

\begin{tabular}{|c|c|c|c|c|}
\hline & MIE & HIIE & $95 \% \mathrm{CI}$ & ES \\
\hline \multicolumn{5}{|l|}{ Boys* } \\
\hline Mean HR $\left(b \cdot \min ^{-1}\right)$ & $122 \pm 9$ & $152 \pm 9$ & 21 to 39 & 3.33 \\
\hline Mean HR (\% HR $\left.\mathrm{HRx}_{\max }\right)$ & $61 \pm 4$ & $76 \pm 3$ & 10 to 19 & 4.24 \\
\hline Mean $\dot{V} \mathrm{O}_{2}\left(\mathrm{~L} \cdot \mathrm{min}^{-1}\right)$ & $1.17 \pm 0.19$ & $1.50 \pm 0.25$ & 0.21 to 0.45 & 1.50 \\
\hline Mean $\dot{V} \mathrm{O}_{2}\left(\% \dot{V} \mathrm{O}_{2 \max }\right)$ & $45 \pm 6$ & $58 \pm 5$ & 9 to 17 & 2.57 \\
\hline RER & $0.92 \pm 0.02$ & $1.10 \pm 0.02$ & 0.15 to 0.20 & 9.00 \\
\hline RPE & $3 \pm 1$ & $6 \pm 2$ & 2 to 5 & 1.90 \\
\hline PACES & $59 \pm 9$ & $64 \pm 8$ & -3 to 12 & 0.59 \\
\hline Work performed $(\mathrm{kJ})$ & $134 \pm 21$ & $134 \pm 21$ & - & - \\
\hline Energy Expenditure (kJ) & $656 \pm 113$ & - & - & - \\
\hline \multicolumn{5}{|l|}{ Girls $^{\dagger}$} \\
\hline Mean HR $\left(\mathrm{b} \cdot \mathrm{min}^{-1}\right)$ & $133 \pm 12$ & $155 \pm 7$ & 15 to 29 & 2.24 \\
\hline Mean HR (\% HR $\left.\mathrm{HR}_{\max }\right)$ & $68 \pm 6$ & $78 \pm 3$ & 8 to 15 & 2.35 \\
\hline Mean $\dot{V} \mathrm{O}_{2}\left(\mathrm{~L} \cdot \mathrm{min}^{-1}\right)$ & $0.99 \pm 0.09$ & $1.24 \pm 0.13$ & 0.18 to 0.32 & 2.24 \\
\hline Mean $\dot{V} \mathrm{O}_{2}\left(\% \dot{V} \mathrm{O}_{2 \max }\right)$ & $53 \pm 6$ & $66 \pm 5$ & 10 to 16 & 2.25 \\
\hline RER & $0.94 \pm 0.03$ & $1.06 \pm 0.6$ & 0.08 to 0.17 & 2.53 \\
\hline RPE & $4 \pm 1$ & $7 \pm 1$ & 3 to 5 & 3.00 \\
\hline PACES & $58 \pm 5$ & $64 \pm 5$ & 1 to 12 & 1.20 \\
\hline Work performed (kJ) & $106 \pm 12$ & $106 \pm 12$ & - & - \\
\hline Energy Expenditure (kJ) & $545 \pm 68$ & - & - & - \\
\hline
\end{tabular}

HR, heart rate; $\dot{V} \mathrm{O}_{2}$, oxygen uptake; MIE, moderate-intensity exercise trial; HIIE, high-intensity exercise trial; 95\% CI = 95\% confidence interval for the true difference; $E S$ $=$ effect size

Data presented as mean \pm SD for MIE and HIIE

$* n=10$ apart from mean HR where $n=9$ due to data loss

${ }^{\dagger} n=10$ apart from mean HR where $n=8$ due to data loss 
Table 3: Postprandial plasma [TAG] and [glucose]

\section{Boys}

TAUC-TAG $\left(\mathrm{mmol} \cdot \mathrm{L}^{-1} \cdot 4 \mathrm{~h}\right)$

IAUC-TAG $\left(\mathrm{mmol} \cdot \mathrm{L}^{-1} \cdot 4 \mathrm{~h}\right)$

TAUC-glucose $\left(\mathrm{mmol} \cdot \mathrm{L}^{-1} \cdot 4 \mathrm{~h}\right)$

IAUC-glucose $\left(\mathrm{mmol} \cdot \mathrm{L}^{-1} \cdot 4 \mathrm{~h}\right)$

Girls

TAUC-TAG $\left(\mathrm{mmol} \cdot \mathrm{L}^{-1} \cdot 4 \mathrm{~h}\right)$

IAUC-TAG $\left(\mathrm{mmol} \cdot \mathrm{L}^{-1} \cdot 4 \mathrm{~h}\right)$

TAUC-glucose $\left(\mathrm{mmol} \cdot \mathrm{L}^{-1} \cdot 4 \mathrm{~h}\right)$

IAUC-glucose $\left(\mathrm{mmol} \cdot \mathrm{L}^{-1} \cdot 4 \mathrm{~h}\right)$

$$
\begin{array}{r}
3.53 \pm 1.54 \\
1.98 \pm 0.83 \\
20.59 \pm 1.00 \\
0.74 \pm 0.92
\end{array}
$$

$$
\begin{array}{r}
3.89 \pm 2.46 \\
2.37 \pm 1.71 \\
20.46 \pm 0.80 \\
0.55 \pm 0.76
\end{array}
$$

$$
\begin{array}{r}
3.36 \pm 1.16 \\
2.01 \pm 0.80 \\
20.98 \pm 1.29
\end{array}
$$$$
0.91 \pm 1.80
$$

$$
\begin{array}{r}
3.35 \pm 1.46 \\
1.57 \pm 0.97 \\
20.23 \pm 1.12 \\
0.67 \pm 1.29
\end{array} \text { * }
$$

$3.46 \pm 1.38$

$2.18 \pm 0.86$

$20.39 \pm 1.22$

$0.92 \pm 1.43$

TAUC, total area under the curve; IAUC, incremental area under the curve; [TAG], plasma triacylglycerol; MIE, moderate-intensity exercise trial; HIIE, high-intensity interval exercise trial.

$*=P<0.05$ for MIE vs CON

${ }^{\dagger}=P<0.05$ for HIIE boys vs girls. 


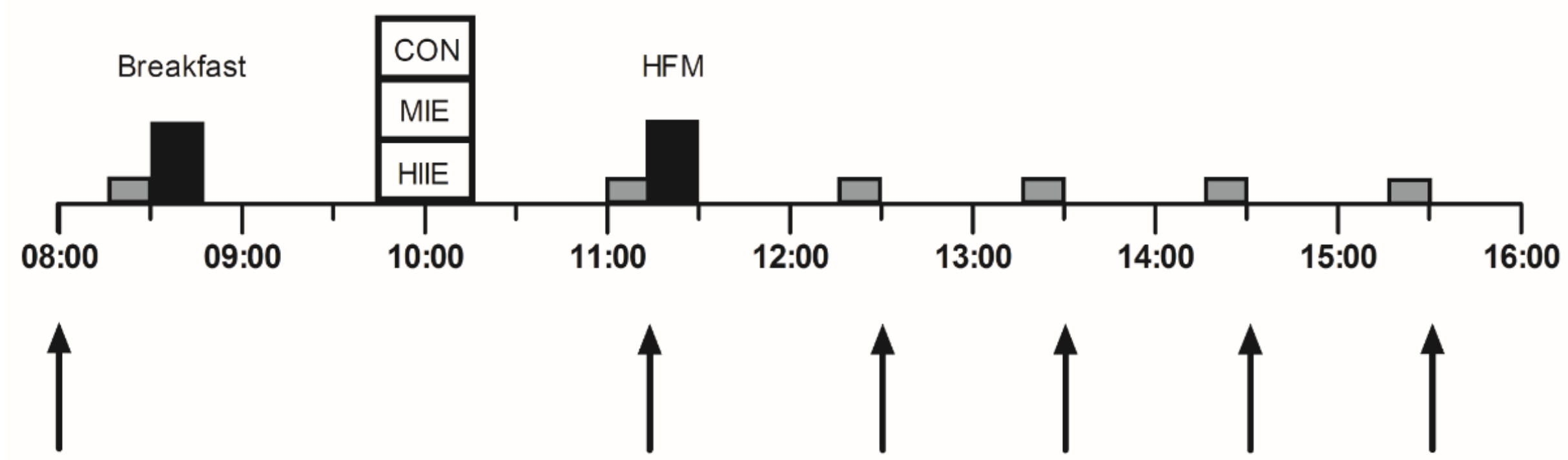

Figure 1 Protocol schematic. 1 = rest; 2 = moderate-intensity exercise; 3 = high-intensity interval exercise. Arrows represent capillary blood samples for plasma [TAG] and glucose; grey boxes represent the assessment of resting metabolic rate and blood pressure; HFM = high fat meal. 
A

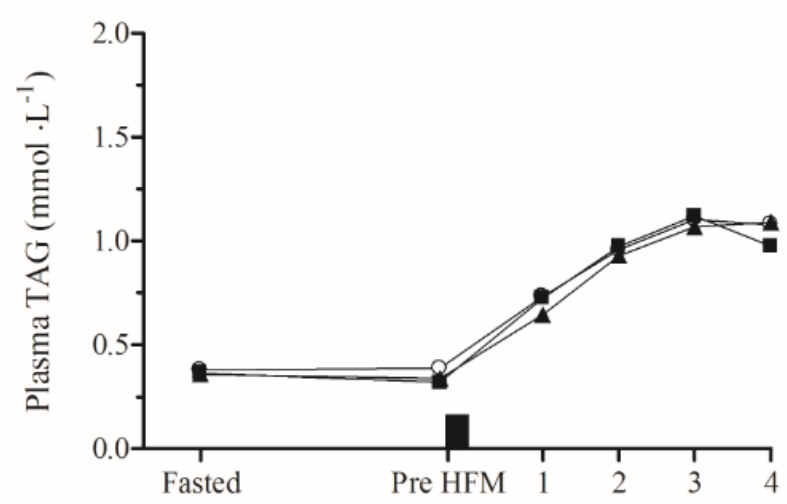

C

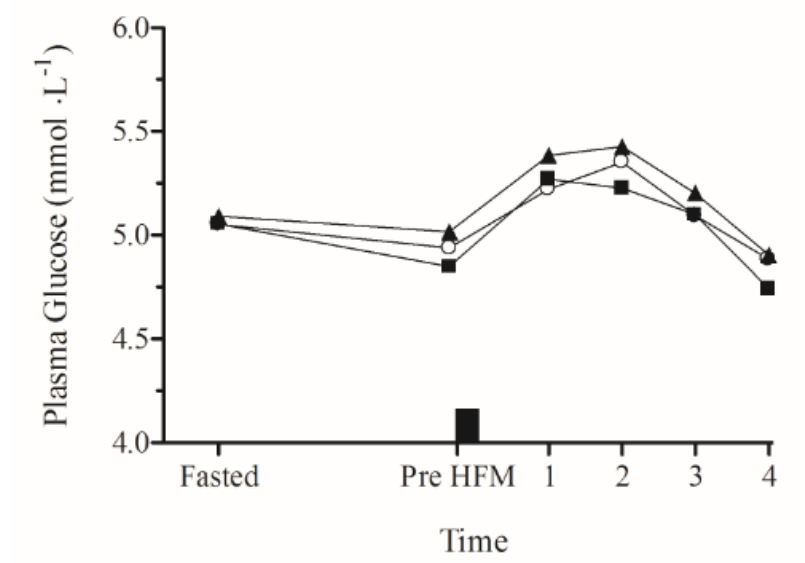

B

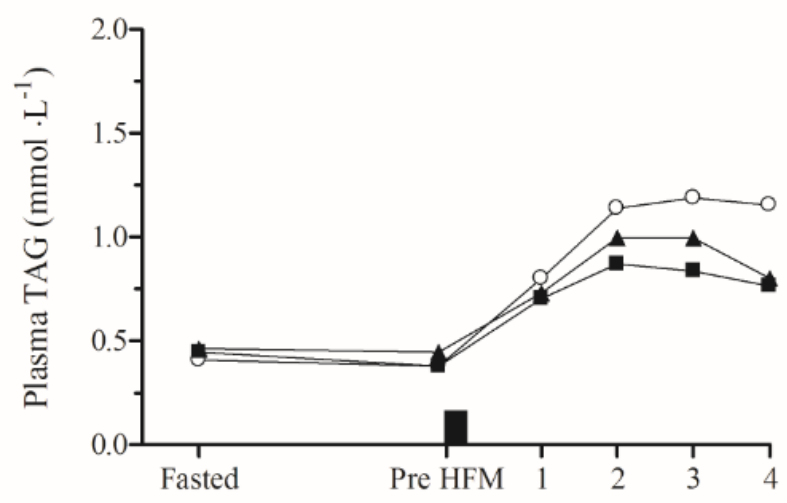

D

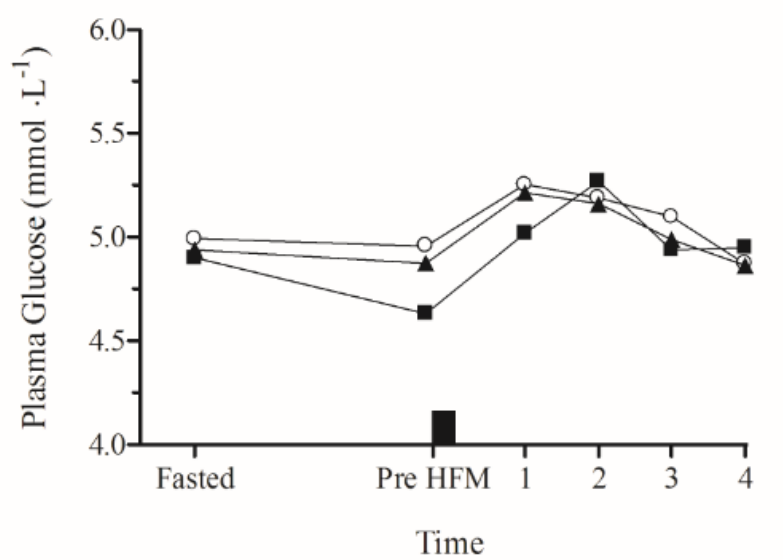

Figure 2 Mean postprandial plasma triacylglycerol ([TAG]) and [glucose] for the control $(\circ)$, moderate- $(\boldsymbol{\Delta})$ and high- $(\boldsymbol{\square})$ intensity exercise conditions for boys $($ A, C) and girls (B, D). Error bars are omitted for clarity. The high fat meal (HFM) is represented by the black rectangle. 

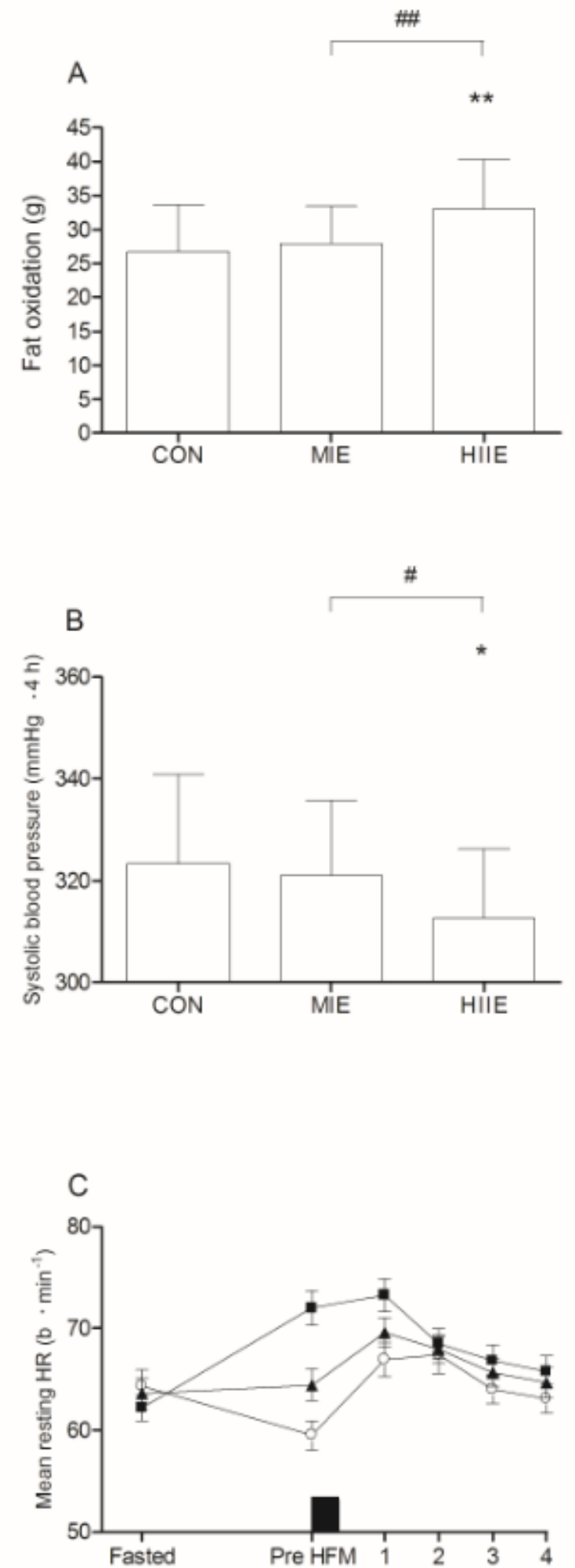

Figure 3 Mean total area under the curves for postprandial fat oxidation (A) and systolic blood pressure vs time (4 hours; B), and heart rate (C) collapsed for the boys and girls $(n=20)$. CON, control trial (O); MIE, moderateintensity exercise trial ( $\Delta$ ); HIIE, high-intensity interval exercise trial ( $\mathbf{\square}){ }^{* *}=P<0.001$ for HIIE vs CON; $*=$ $P<0.05$ for HIIE vs CON; ${ }^{\# \#}=P<0.001$ for HIIE vs MIE; ${ }^{\#}=P<0.05$ for HIIE vs MIE. The high fat meal (HFM) is represented by the black rectangle. Error bars describe the standard deviation. 